\title{
Phthisis bulbi and retinal capillary haemangioma associated with papilloedema due to recurrent bilateral cerebellar haemangioblastomas - A case of Von Hippel Lindau syndrome
}

\author{
Muthukrishnan Vallinayagam ${ }^{1, *}$, Srikanth $\mathrm{K}^{2}$, Tintu Susan Joy ${ }^{3}$, Koushik Shivakumar ${ }^{4}$ \\ ${ }^{\mathbf{1}}$ Associate Professor, ${ }^{2}$ Professor, ${ }^{3}$ Senior Resident, ${ }^{4}$ Junior Resident, Dept. of Ophthalmology, Mahatma Gandhi Medical College and \\ Research Institute, Puducherry, India
}

*Corresponding Author:

Email: muthu2308@yahoo.co.in

\begin{abstract}
Von Hippel Lindau (VHL) syndrome is an autosomal dominant condition caused by mutation of VHL gene. It is characterized by vascular tumors of eye, central nervous system, visceral cysts and tumors. Here we report a case of VHL in a young male patient who presented with phthisis bulbi in the right eye and retinal capillary haemangioma in the left eye. Systemic features of raised intracranial pressure was present along with bilateral abduction deficit and florid disc edema in the left eye. There was a polypoidal reddish mass in the inferotemporal quadrant, with a dilated draining vein, indicating retinal capillary hemangioma. The phthisical right eye was speculated to result from untreated retinal capillary hemangioma, culminating in exudative retinal detachment. Urinary Vanillyl Mandelic Acid suggested pheochromocytoma. Imaging modalities revealed multiple cerebellar hemangioblastomas, pheochromocytoma and pancreatic cysts. Screening of family members revealed no evidence of systemic manifestations, suggesting the possibility of sporadic mutation. Retinal capillary hemangioma is the commonest and earliest manifestation of VHL. The ocular complications include intraretinal exudation and exudative retinal detachment, secondary angiomatosis, epiretinal membrane, vitreous hemorrhage, retinal neovascularization, tractional retinal detachment and macular ectopia. Our case report emphasizes that retinal examination in patients with suspected VHL is not only a screening tool for the presence of capillary hemangioma but also for regular monitoring and prevention of vision threatening complications.
\end{abstract}

Keywords: Von Hippel Lindau (VHL) syndrome, Retinal capillary hemangioma, Cerebellar hemangioblastomas, Pheochromocytoma.

\section{Introduction}

Von Hippel Lindau syndrome (VHL) is a dominantly inherited phacomatosis with an incidence of 1 in 36,000 to 1 in $53,000 .{ }^{1}$ It is caused by mutation of VHL gene (tumor suppressor gene) in chromosome $3 \mathrm{p} 25-\mathrm{p} 26 .^{2}$ It is characterized by multiple tumors of the central nervous system, retina, kidney, pancreas, adrenals, and inner ear. ${ }^{1}$ Retinal capillary hemangioma is the earliest and most frequent manifestation of VHL with a mean age of presentation at 25 years of age. Retinal capillary hemangiomas are usually found in peripheral, midperipheral or juxtapapillary retina. ${ }^{1,2}$ It is an uncommon benign tumor which may remain asymptomatic or may progressively enlarge and cause sight threatening complications. VHL is a lethal disease with renal cell carcinoma and central nervous system hemangioblastomas being the leading causes of death in the majority of cases. ${ }^{3}$ Early diagnosis, follow up and prompt treatment of retinal capillary hemangioma is imperative for preventing vision threatening complications like exudative retinal detachment.

\section{Case Report}

A 27 year old male patient presented with severe headache and transient blurring of vision in the left eye. The headache was associated with episodes of nausea, vomiting, palpitation and sweating. There was history of gradual and painless loss of vision in the right eye seven years back. The patient denied history of ocular trauma. The past history was discerned by a neurosurgical procedure eight years back, for a brain tumor. There was no history of similar ailments in the family members.
Systemic examination revealed hypertensive spikes on regular 24 hour monitoring. There was no obvious organomegaly. The visual acuity was recorded as absent perception of light in right eye and 6/9 in the left eye. There was limitation of abduction in both eyes. (Fig. 1 \& 2) Complicated cataract with posterior synechiae was noted in the right eye with no evidence of active anterior uveitis. The cataractous lens obscured visualization of fundus. The anterior segment evaluation of the left eye was within normal limits.

Fundus examination of left eye showed florid disc edema. (Fig. 3) There was a polypoidal reddish mass in the inferotemporal quadrant, with a dilated draining vein, adjacent to the equator, suggestive of retinal capillary hemangioma. (Fig. 4) Ultrasonography (B scan) in the right eye revealed features suggestive of old exudative retinal detachment. FFA was deferred due to deranged renal parameters. CECT abdomen revealed a well defined solid lesion in right adrenal gland with vivid contrast enhancement suggesting pheochromocytoma. (Fig. 5) Multiple pancreatic cysts were visualized. (Fig. 6) MRI brain showed multiple enhancing lesions in bilateral cerebellar hemispheres suggestive of multiple hemangioblastomas. (Fig. 7) There was a cyst with mural nodule in posterior cranial fossa causing tonsillar herniation, obstructive hydrocephalus and mass effect over brain stem. (Fig. 8) Urinary Vanillyl Mandelic Acid suggested the presence of pheochromocytoma. A screening of family members was performed due to the dominant inheritance of the tumor and none were found to be affected. 
In view of specific conglomerate association of retinal capillary hemangioma, cerebellar tumor, suprarenal mass and pancreatic cyst, a diagnosis of Von Hippel Lindau syndrome was made. Patient underwent neurosurgical intervention for excision of the cerebellar tumor. Since the retinal capillary hemangioma was a small lesion away from the posterior pole, patient was advised three monthly follow up visits.

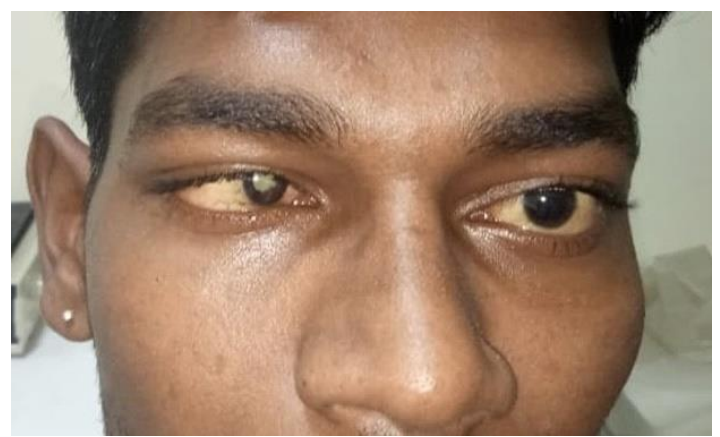

Fig. 1: Restriction of abduction in (LE)

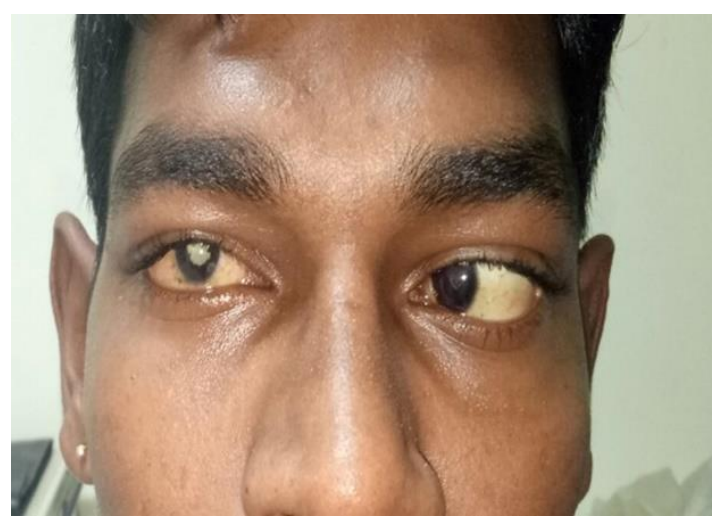

Fig. 2: Restriction of abduction in (RE)

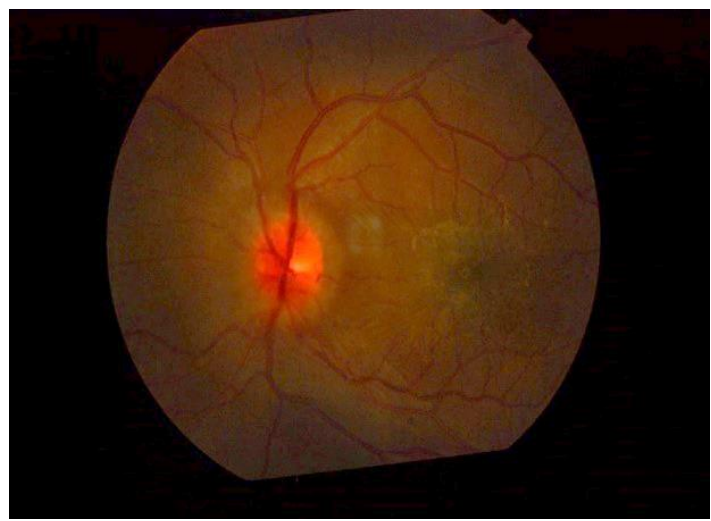

Fig. 3: (LE) Disc edema

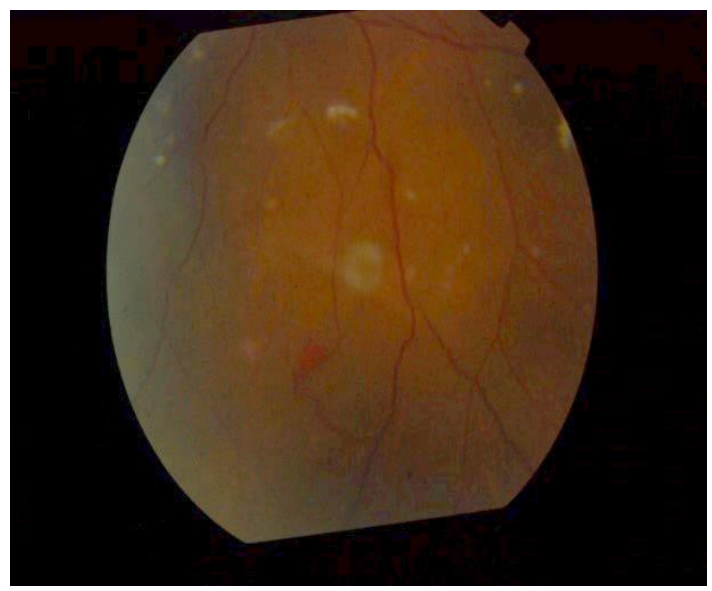

Fig. 4: (LE) Retinal capillary hemangioma in inferotemporal quadrant

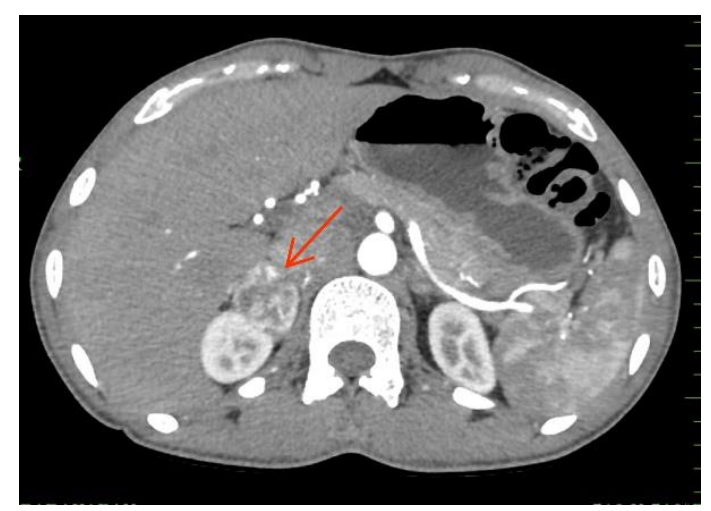

Fig. 5: CECT abdomen showing right adrenal mass

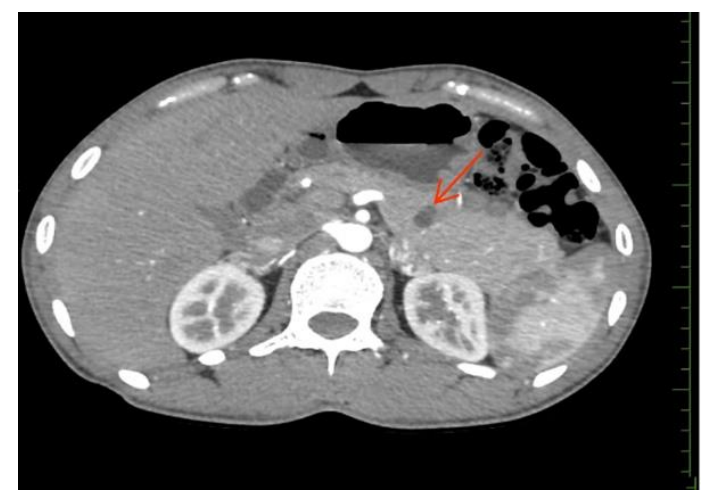

Fig. 6: CECT abdomen showing pancreatic cyst 


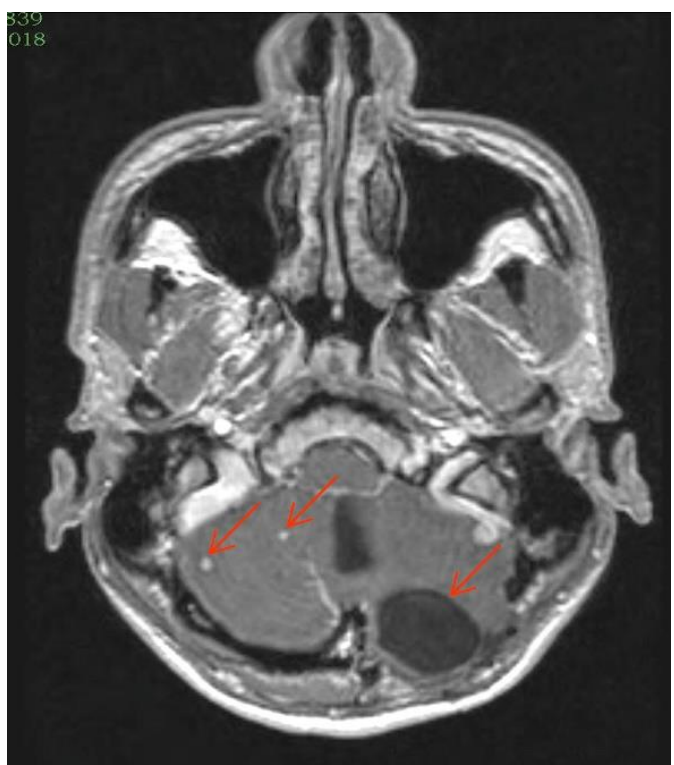

Fig. 7: MRI brain showing multiple hemangioblastoma

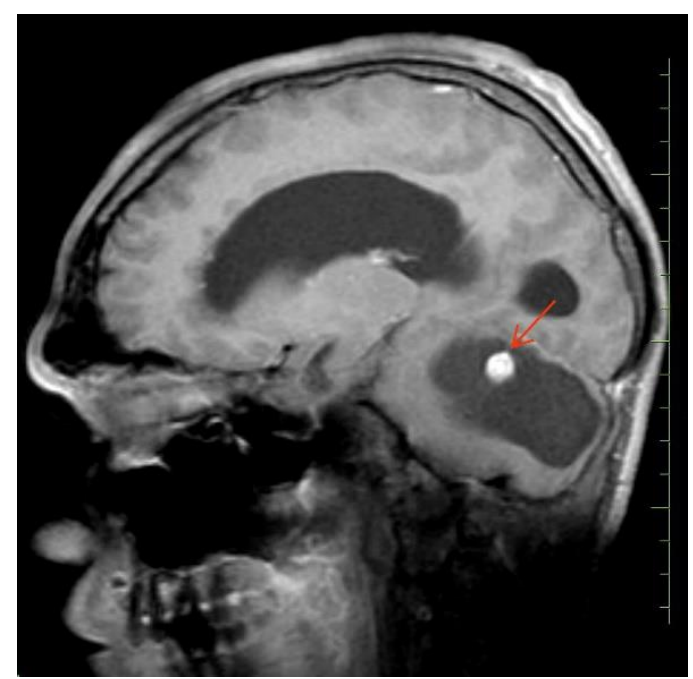

Fig. 8: MRI brain (sagittal view) showing a cyst with mural nodule in posterior cranial fossa

\section{Discussion}

Arvid Lindau, a swedish pathologist first established the association of retinal capillary hemangioma with cerebellar hemangioblastoma. ${ }^{4}$ Melmon and Rosen proposed the clinical diagnostic criteria for Von Hippel Lindau syndrome. ${ }^{4,5}$ The diagnosis of VHL was considered in this patient due to the presence of retinal capillary hemangioma, pancreatic cyst, pheochromocytoma and cerebellar hemangioblastoma. A study on 103 patients with retinal manifestations of VHL showed that $75 \%$ patients had CNS hemangioblastoma, $51 \%$ had renal cell carcinoma, $20 \%$ had pheochromocytoma and $16 \%$ had pancreatic lesions. ${ }^{1}$ Screening of family members in this case revealed no evidence of systemic manifestations of VHL, suggesting the possibility of sporadic mutation. $73 \%$ of VHL mutations are inherited and $17 \%$ are sporadic. ${ }^{2}$ Since majority of cases are inherited, screening of family members is mandatory.
A study on 205 patients with ocular VHL found that $55.1 \%$ patients had bilateral retinal hemangioblastoma and $44.9 \%$ patients had unilateral involvement. ${ }^{4} 56.2 \%$ of patients had multiple retinal lesions at the time of presentation. ${ }^{4}$ In another study, $67.8 \%$ of patients with VHL had retinal angiomas and the median age was 31 years. ${ }^{6}$ Retinal angiomas are most commonly found in the superotemporal quadrant (38.6\%), followed by inferotemporal quadrant $(27.3 \%){ }^{6} \mathrm{~A}$ solitary lesion was present in the inferotemporal quadrant of a 27 year old patient in this case.

The natural course of development of retinal capillary hemangioma can be categorized into several stages. Stage 1 retinal capillary hemangioma is a small peripheral pink spot without any feeder vessels or vascular channels. Stage 2 tumor is a small slightly elevated nodule with only a prominent draining vein. Stage 3 tumor has a classical appearance with prominent feeder vessels. Stage 4 demonstrates partial exudative retinal detachment and in stage 5 , a total exudative retinal detachment. ${ }^{7}$ Literature review shows that $55 \%$ of VHL patients with retinal hemangioblastomas were asymptomatic at the time of presentation. ${ }^{2}$ Retinal capillary hemangioma may remain stable, regress or progressively enlarge leading to exudative retinal detachment, uveitis, glaucoma and phthisis in the later stages. ${ }^{3}$ This case portays an asymptomatic $\mathrm{RCH}$ categorized as stage 2 .

The ocular complications in VHL can result from intraretinal exudation and exudative retinal detachment, secondary angiomatosis, epiretinal membrane, vitreous hemorrhage, retinal neovascularization, tractional retinal detachment and macular ectopia. ${ }^{6,8} \mathrm{~A}$ case study reported permanent visual loss in $26.2 \%$ and the maximum risk of visual loss to occur prior to 20 years of age. ${ }^{6}$ This scenario closely resembles to that described in this patient.

The goal of treatment in retinal capillary hemangioma is to preserve vision and to salvage the eye. ${ }^{8}$ Stage 1 and stage 2 tumors require vigilant observation. Treatment is indicated for stage 3 and beyond. Complications like tractional or exudative retinal detachment may occur following treatment. ${ }^{7}$ In a study on ocular angiomatosis in VHL, exudative retinal detachment was observed in $15.5 \%$ patients. ${ }^{6}$ Small peripheral retinal tumors can be treated with laser whereas larger tumors may require cryotherapy. The other modalities like brachytherapy or external beam radiotherapy can be used when standard therapy fails to cause regression. Surgical resection is considered only for large stage retinal hemangiomas. ${ }^{8}$

Screening of family members is vital as the syndrome has a strong genetic basis. Prognosis of patients with VHL has improved in recent years due to modern imaging techniques which aid in early diagnosis. With increasing survival rates in VHL, the prevalence of blinding ocular disease has also escalated proportionately. ${ }^{1}$ Exudative retinal detachment can be prevented by early diagnosis and prompt treatment. Retinal examination in VHL is mandatory for screening and monitoring of early lesions. 


\section{Conclusion}

Ocular examination plays an important role in early diagnosis and screening of patients with VHL which reduces the morbidity and mortality associated with the disease. Our case report emphasizes that retinal examination in suspected VHL patients is not only a screening tool for capillary hemangioma but also for regular monitoring of retinal lesions for vision threatening complications. A vigilant follow up is imperative.

\section{Conflict of Interest: Nil.}

\section{References}

1. Dollfus H, Massin P, Taupin P, Nemeth C, Amara S, Giraud S, Béroud C, Dureau P, Gaudric A, Landais P, Richard S. Retinal hemangioblastoma in von Hippel-Lindau disease: a clinical and molecular study. Invest Ophthalmol Vis Sci. 2002;43(9):3067-3074.

2. Niemelä M, Lemeta S, Sainio M, Rauma S, Pukkala E, Kere J, Böhling T, Laatikainen L, Jääskeläinen J, Summanen P. Hemangioblastomas of the retina: impact of von HippelLindau disease. Invest Ophthalmol Vis Sci. 2000;41(7):19091915.

3. Neumann HP, Finger PT. Von Hippel-Lindau Syndrome. Retina Today. 2010

4. Chew EY. Ocular manifestations of von Hippel-Lindau disease: clinical and genetic investigations. Trans Am Ophthalmol Soc. 2005;103:495.
5. Mihmanli I, Albayram MS, Kantarci F, Adaletli I, Islak C, Altug A. Persistent Hyperplastic Primary Vitreous and von Hippel-Lindau Disease. J Ultrasound Med. 2002;21(5):565568.

6. Webster AR, Maher ER, Moore AT. Clinical characteristics of ocular angiomatosis in von Hippel-Lindau disease and correlation with germline mutation. Arch Ophthalmol. 1999;117(3):371-378.

7. Wittebol-Post D, Hes FJ, Lips CJ. The eye in von HippelLindau disease. Long-term follow-up of screening and treatment: recommendations. J Internal Med. 1998;243(6):555561.

8. Kase S, Ishida S. Retinal Capillary Hemangioma in Von Hippel Lindau disease: current concept, diagnosis and managements. J Transl Med Epidemiol. 2(1):1010.

How to cite this article: Vallinayagam M, Srikanth K, Joy T. S, Shivakumar K. Phthisis bulbi and retinal capillary haemangioma associated with papilloedema due to recurrent bilateral cerebellar haemangioblastomas - A case of Von Hippel Lindau syndrome. Int $\mathbf{J}$ Ocul Oncol Oculoplasty. 2018;4(4):174-177. 